International Journal of Bifurcation and Chaos, Vol. 9, No. 1 (1999) 215-219

(C) World Scientific Publishing Company

\title{
GENERALIZED SYNCHRONIZATION OF CHAOS VIA LINEAR TRANSFORMATIONS
}

\author{
TAO YANG and LEON O. CHUA \\ Department of Electrical Engineering and Computer Sciences, \\ University of California at Berkeley, \\ Berkeley, CA 94720, USA
}

Received April 18, 1998; Revised July 13, 1998

\begin{abstract}
Generalized synchronization (GS) of two chaotic systems is a generalization of identical synchronization. Usually, the manifold of GS is much more complex than the driven system and the driving system. In this paper, we study a special case of GS in which the synchronization manifold is linear (linear GS for short). In a theorem, we present the necessary and sufficient conditions under which a linear GS can be achieved between two chaotic systems. In particular, we study the linear GS of two Chua's circuits.
\end{abstract}

\section{Introduction}

In view of the potential applications of chaotic synchronization [Yang \& Chua, 1996a, 1996b; Yang et al., 1997], many different chaotic synchronization methods have been developed recently. Among them are: Identical synchronization (synchronization in the usual sense) [Carroll \& Pecora, 1991] impulsive synchronization [Yang \& Chua, 1997a, 1997b; Yang et al., 1998] and general synchronization (GS) [Yang \& Chua, 1996b; Hunt et al., 1997]. The so-called generalized synchronization can give much richer dynamics than identical ones. Even some desynchronized cases (in the usual sense) due to the parameter mismatches [Wu et al., 1996], distortions in transmitting channels [Chua et al., 1996] and some other distortions can be regarded as GS. Applications of GS may be wider or more practical than those of identical synchronization because there always exist parameter mismatches and distortions in the physical world. For example, one recent application of GS is the design of a channelindependent chaotic secure communication system [Yang \& Chua, 1996b]. In this paper, we present the theoretical results which give the conditions for a specific kind of GS whose synchronizing manifold is linear (we call it linear GS for short). We use a standard chaotic system, Chua's circuit, to demonstrate the validity of our theoretical results.

\section{Linear Generalized Synchronization}

Consider two dynamical systems

$$
\begin{cases}\dot{\mathbf{x}}=f(\mathbf{x}) & \leftarrow \text { driving system } \\ \dot{\mathbf{y}}=g(\mathbf{y}, h(\mathbf{x})) & \leftarrow \text { driven system }\end{cases}
$$

where $\mathbf{x} \in \mathbf{R}^{n}, \mathbf{y} \in \mathbf{R}^{m}, h: \mathbf{R}^{n} \mapsto \mathbf{R}^{m}$ is an arbitrary function.

Definition 1. Generalized synchronization (GS) [Yang \& Chua, 1996b]

The two systems in (1) are said to be in a state of generalized synchronization, henceforth referred to as GS, if there exist a transformation $H: \mathbf{R}^{n} \mapsto$ $\mathbf{R}^{m}$, a manifold $M=\{(\mathbf{x}, \mathbf{y}) \mid \mathbf{y}=H(\mathbf{x})\}$, and a set $B \subset \mathbf{R}^{n} \times \mathbf{R}^{m}$ with $M \subset B$ such that all trajectories of (1) with initial conditions in $B$ approach $M$ as $t \rightarrow \infty$.

Remark. Synchronization in the normal sense (identical synchronization) is a special case of GS with $m=n$, and $H(\mathbf{x})=\mathbf{x}$. 
Assume that a chaotic system can be decomposed into two parts ${ }^{1}$

$$
\dot{\mathbf{x}}=A \mathbf{x}+\psi(\mathbf{x})
$$

where $A$ is an $n \times n$ constant matrix and $\psi: \mathbf{R}^{n} \mapsto$ $\mathbf{R}^{n}$. Assume that we transmit the signal $\psi(\mathbf{x})$ to the driven system and consider the following unidirectional synchronization scheme:

$$
\left\{\begin{array}{l}
\dot{\mathbf{x}}=A \mathbf{x}+\psi(\mathbf{x}) \quad \leftarrow \text { driving system } \\
\dot{\mathbf{y}}=A \mathbf{y}+\Lambda \psi(\mathbf{x}) \quad \leftarrow \text { driven system }
\end{array}\right.
$$

Theorem 1. If the matrix $\Lambda$ commutes with $A$, then the two dynamic systems in Eq. (3) are in a state of GS via the following GS transformation

$$
\mathbf{y}(\infty)=H(\mathbf{x})=\Lambda \mathbf{x}
$$

if and only if $A$ is negative definite.

Proof. Let $\mathbf{z}=\mathbf{y}-\Lambda \mathbf{x}$, then the stability of the GS between the two dynamical systems in Eq. (3) via the GS transformation $\mathbf{y}=H(\mathbf{x})=\Lambda \mathbf{x}$ is equivalent to that of the origin of the following system:

$$
\begin{aligned}
\dot{\mathbf{z}} & =[A \mathbf{y}+\Lambda \psi(\mathbf{x})]-[\Lambda A \mathbf{x}+\Lambda \psi(\mathbf{x})] \\
& =A \mathbf{y}-\Lambda A \mathbf{x} \\
& =A(\mathbf{y}-\Lambda \mathbf{x})(\text { since } \Lambda \text { commutes with } A) \\
& =A \mathbf{z} .
\end{aligned}
$$

$\mathbf{z}=\mathbf{0}$ is asymptotically stable if and only if $A$ is negative definite.

Remark. When the GS is achieved, the state variables of the driving system and the driven system are connected by a linear transformation. This is why we call this kind of GS as linear GS.

The matrices which commute with an $n \times n$ matrix $A$ are solutions of the following matrix equation:

$$
A \mathbf{X}=\mathbf{X} A
$$

where $\mathbf{X}$ is an $n \times n$ matrix variable. Since the above equation has an infinite number of solutions, this provides us with several methods to construct linear GS between two chaotic systems. In the following sections, we present some results to demonstrate this fact.

\section{Linear Generalized Synchronization of Two Chua's Circuits}

In this section we study the linear GS of two Chua's circuits. A Chua's circuit [Madan, 1993; Chua, 1994], which consists of two linear capacitors $C_{1}$ and $C_{2}$, a linear inductor $L$, two linear resistors $R$ and $R_{0}$, and a piecewise-linear negative resistor called a Chua's diode, is described by the following state equation:

$$
\left\{\begin{aligned}
\frac{d v_{1}}{d t} & =\frac{1}{C_{1}}\left[G\left(v_{2}-v_{1}\right)-f\left(v_{1}\right)\right] \\
\frac{d v_{2}}{d t} & =\frac{1}{C_{2}}\left[G\left(v_{1}-v_{2}\right)+i_{3}\right] \\
\frac{d i_{3}}{d t} & =-\frac{1}{L}\left[v_{2}+R_{0} i_{3}\right]
\end{aligned}\right.
$$

where $G=1 / R$ and $f(\cdot)$ is the piecewise-linear characteristics defined by

$$
f\left(v_{1}\right)=G_{b} v_{1}+\frac{1}{2}\left(G_{a}-G_{b}\right)\left(\left|v_{1}+E\right|-\left|v_{1}-E\right|\right)
$$

where $E$ is the breakpoint voltage of the Chua's diode. The corresponding circuit is shown in Fig. 1. The block diagram of the circuit implementation of Chua's circuit and the parameters can be found in [Kennedy, 1992].

Let $\mathbf{x}=\left(\begin{array}{lll}v_{1} & v_{2} & i_{3}\end{array}\right)^{T}$, then Chua's circuit can be decomposed as

$$
\begin{gathered}
A=\left(\begin{array}{ccc}
-\frac{G}{C_{1}} & -\frac{G}{C_{1}} & 0 \\
\frac{G}{C_{2}} & -\frac{G}{C_{2}} & \frac{1}{C_{2}} \\
0 & -\frac{1}{L} & -\frac{R_{0}}{L}
\end{array}\right), \\
\Psi(\mathbf{x})=\left(\begin{array}{c}
\psi_{1}(\mathbf{x}) \\
\psi_{2}(\mathbf{x}) \\
\psi_{3}(\mathbf{x})
\end{array}\right)=\left(\begin{array}{c}
-\frac{1}{C_{1}} f\left(v_{1}\right) \\
0 \\
0
\end{array}\right) .
\end{gathered}
$$

\footnotetext{
${ }^{1}$ It should be noticed that this decomposition is very general in the sense that for a given chaotic system $\dot{\mathbf{x}}=f(\mathbf{x})$ and a prescribed matrix $A$, we can always have: $\dot{\mathbf{x}}=A \mathbf{x}+(f(\mathbf{x})-A \mathbf{x})$. If we let $\psi(\mathbf{x})=(f(\mathbf{x})-A \mathbf{x})$ we then get this kind of decomposition.
} 


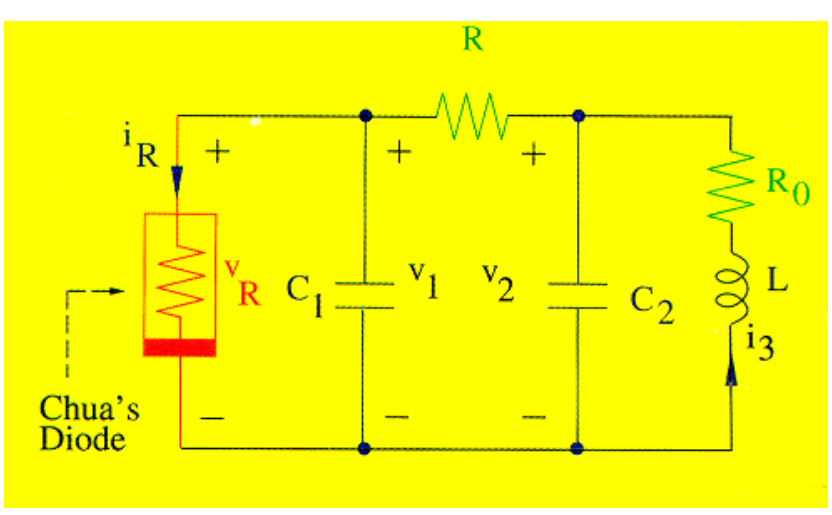

(a)

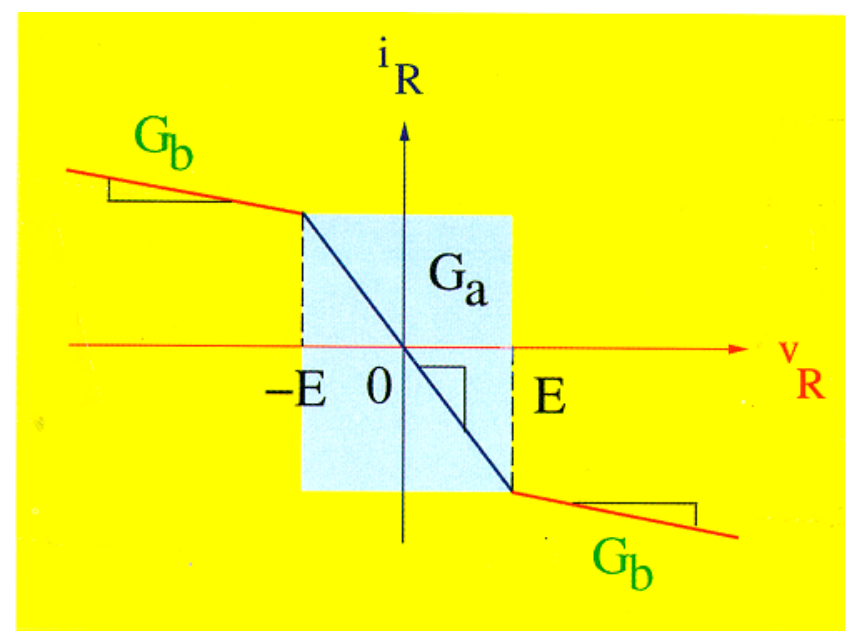

(b)

Fig. 1. (a) Block diagram of Chua's circuit. (b) Piecewiselinear characteristic of Chua's diode.

Since $\psi_{2}(\mathbf{x})=\psi_{3}(\mathbf{x})=0$ it is not necessary to assign them to the driven system. What we needed to send is the signal $\psi_{1}(\mathbf{x})=-1 / C_{1} f\left(v_{1}\right)$. Since $C_{1}$ in our simulations is considered as a constant, we only need to send the signal $f\left(v_{1}\right)$, which is the current through the Chua's diode in the driving system.

In this section, we present two simulation results. The parameters of Chua's circuits are chosen as follows: $C_{1}=5.56 \mathrm{nF}, C_{2}=50 \mathrm{nF}, G=0.7 \mathrm{mS}$, $L=7.14 \mathrm{mH}, G_{a}=-0.8 \mathrm{mS}, G_{b}=-0.5 \mathrm{mS}$, $E=1$ and $R_{0}=0$. Under these conditions, the eigenvalues of $A$ are all located in the open left-hand plane. The fourth-order Runge-Kutta method with $5 \mu$ s fixed step size is used. The initial conditions for the driving Chua's circuit and the driven Chua's circuit are given respectively by $\left(v_{1}(0), v_{2}(0), i_{3}(0)\right)=(-0.2 \mathrm{~V},-0.2 \mathrm{~V},-1 \mathrm{~mA})$, and $\left(\tilde{v}_{1}(0), \tilde{v}_{2}(0), \tilde{i}_{3}(0)\right)=(-0.2 \mathrm{~V},-0.2 \mathrm{~V}, 1 \mathrm{~mA})$.

\subsection{Simulation 1}

In this simulation, we let

$$
\Lambda=\left(\begin{array}{lll}
\lambda & 0 & 0 \\
0 & \lambda & 0 \\
0 & 0 & \lambda
\end{array}\right)
$$

where $\lambda \neq 0$. Observe that $\Lambda A=A \Lambda$. This is a very simple case that had been used in [Yang \& Chua, 1996b] for building a channel independent chaotic secure communication system. The driven Chua's circuit is given by

$$
\left\{\begin{array}{l}
\frac{d \tilde{v}_{1}}{d t}=\frac{1}{C_{1}}\left[G\left(\tilde{v}_{2}-\tilde{v}_{1}\right)-\lambda f\left(v_{1}\right)\right] \\
\frac{d \tilde{v}_{2}}{d t}=\frac{1}{C_{2}}\left[G\left(\tilde{v}_{1}-\tilde{v}_{2}\right)+\tilde{i}_{3}\right] \\
\frac{d \tilde{i}_{3}}{d t}=-\frac{1}{L}\left[\tilde{v}_{2}+R_{0} \tilde{i}_{3}\right]
\end{array} .\right.
$$

The simulation result is shown in Fig. 2(a). We choose $\lambda=0.5$. Observer that the attractor of the driven system is a scaled version of that of the driving system with a scale factor 0.5 .

\subsection{Simulation 2}

In this simulation, we choose

$$
\Lambda=A=\left(\begin{array}{ccc}
-\frac{G}{C_{1}} & -\frac{G}{C_{1}} & 0 \\
\frac{G}{C_{2}} & -\frac{G}{C_{2}} & \frac{1}{C_{2}} \\
0 & -\frac{1}{L} & -\frac{R_{0}}{L}
\end{array}\right) .
$$

Observe that $\Lambda A=A \Lambda=A^{2}$. In this case, the driven Chua's circuit is given by

$$
\left\{\begin{array}{l}
\frac{d \tilde{v}_{1}}{d t}=\frac{1}{C_{1}}\left[G\left(\tilde{v}_{2}-\tilde{v}_{1}\right)\right]+\frac{G}{C_{1}^{2}} f\left(v_{1}\right) \\
\frac{d \tilde{v}_{2}}{d t}=\frac{1}{C_{2}}\left[G\left(\tilde{v}_{1}-\tilde{v}_{2}\right)+\tilde{i}_{3}\right]-\frac{G}{C_{1} C_{2}} f\left(v_{1}\right) . \\
\frac{d \tilde{i}_{3}}{d t}=-\frac{1}{L}\left[\tilde{v}_{2}+R_{0} \tilde{i}_{3}\right]
\end{array}\right.
$$

If the GS between the driving and the driven Chua's circuits is achieved, the following relations should be satisfied. 


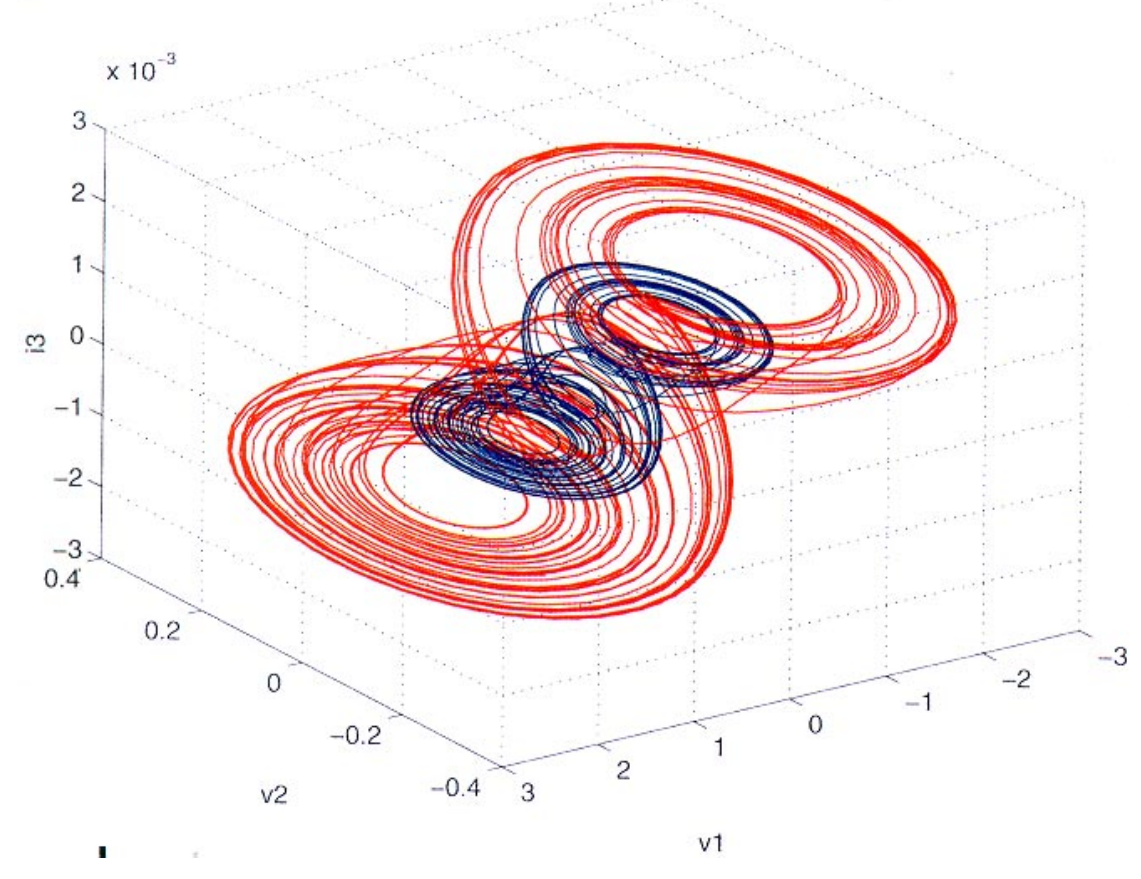

(a)

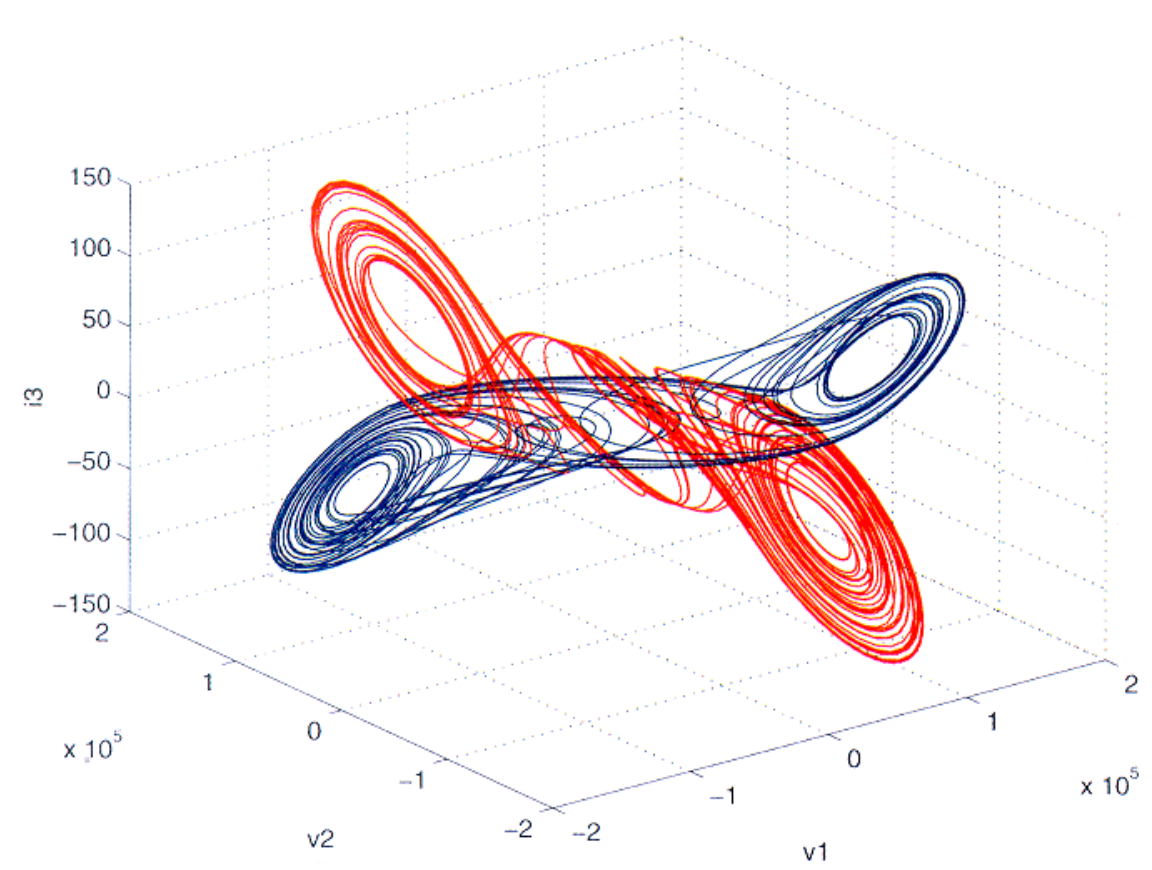

(b)

Fig. 2. Linear GS of two Chua's circuits. The attractor of the driving system is shown in red and that of the driven system is shown in blue. (a) Result of simulation 1. (b) Result of simulation 2.

$$
\begin{aligned}
& \tilde{v}_{1}=-\frac{G}{C_{1}} v_{1}+\frac{G}{C_{1}} v_{2} \\
& \tilde{v}_{2}=\frac{G}{C_{2}} v_{1}-\frac{G}{C_{2}} v_{2}+\frac{1}{C_{2}} i_{3} \quad \tilde{i}_{3}=i_{3} / L
\end{aligned}
$$

The simulation result is shown in Fig. 2(b). The attractor of the driving system is scaled by a constant vector $\left(10^{5}, 10^{4}, 10^{5}\right)$. Although the shapes of these two attractors are different, the linear transformation of the GS in Eq. (14) is satisfied. 


\section{Conclusions}

In this paper, we present the theoretical results on linear GS between two chaotic systems. We also develop a method for decomposing chaotic systems in a special manner such that the linear GS can be achieved. Especially, we use Chua's circuits to demonstrate the validity of the theoretical results. The theoretical results can be used to design proper linear GS between two chaotic systems. They can also be used to interpret the behavior of the driven system. We observed that GS is a very robust phenomenon to parameter mismatch and noise. The potential application of GS to channelindependent chaotic secure communication was presented in [Yang \& Chua, 1996b].

\section{Acknowledgment}

This work is supported in part by the Office of Naval Research under grant numbers N00014-97-10463 and N00014-96-1-0753.

\section{References}

Carroll, T. L. \& Pecora, L. M. [1991] "Synchronizing chaotic circuits," IEEE Trans. Circuits Syst. 38, 453-456.

Chua, L. O. [1994] "Chua's circuit - An overview ten years later," J. Circuit Syst. Comput. 4(2), 117-159.

Chua, L.O., Yang, T., Zhong, G. Q. \& Wu, C. W. [1996] "Synchronization of chua's circuits with time-varying channels and parameters," IEEE Trans. Circuits Syst. I. Fundamental Theor. Appl. 43(10), 862-868.
Hunt, B. R., Ott, E. \& Yorke, J. A. [1997] "Differentiable generalized synchronization of chaos," Phys. Rev. E55(4), 4029-4034.

Kennedy, M. P. [1992] "Robust OP AMP realization of Chua's circuit," Frequenz 46(3,4), 66-80.

Madan, R. N. [1993] Chua's Circuit: A Paradigm for Chaos (World Scientific, Singapore).

Wu, C. W., Yang, T. \& Chua, L. O. [1996] "On adaptive synchronization and control of nonlinear dynamical systems," Int. J. Bifurcation and Chaos 6(3), 455-471.

Yang, T. \& Chua, L. O. [1996] "Secure communication via chaotic parameter modulation," IEEE Trans. Circuits Syst. I. Fundamental Theor. Appl. 43(9), 817-819.

Yang, T. \& Chua, L. O. [1996] "Channel-independent chaotic secure communication," Int. J. Bifurcation and Chaos 6(12B), 2653-2660.

Yang, T., Wu, C. W. \& Chua, L. O. [1997] "Cryptography based on chaotic systems," IEEE Trans. Circuits Syst. I. Fundamental Theor. Appl. 44(5), 469-472.

Yang, T. \& Chua, L. O. [1997] "Impulsive control and synchronization of nonlinear dynamical systems and application to secure communication," Int. J. Bifurcation and Chaos 7(3), 645-664.

Yang, T. \& Chua, L. O. [1997] "Impulsive stabilization for control and synchronization of chaotic systems: Theory and application to secure communication," IEEE Trans. Circuits Syst. I: Fundamental Theor. Appl. 44(10), 976-988.

Yang, T., Suykens, J. A. K. \& Chua, L. O. [1998] "Impulsive control of nonautonomous chaotic systems using practical stabilization," Int. J. Bifurcation and Chaos 8(7), 1557-1564. 